\title{
EDITORIAL
}

\section{PERSONALIZED MEDICINE IN PSYCHIATRY}

\author{
Kumar Anil - Shukla S. R. P.
}

In medicine knowledge of the disease and an understanding of its mechanisms are required. In Psychiatry we, are dealing with individual human being as a whole the uniqueness of each person makes him / her as an individual in his / her own SELF therefore the course of the disease in response to treatment vary from one subject to another due to differential in; genetic, enzymatic and metabolic factors (Macher Jean Paul 2009). Psychiatry deals with mind which is not an anatomical organ but a treasured perception of an individual where in his / her individuality lies. Precisely, for this reason mental health professionals come across ample opportunities for conscious as well as conscientious learning to understand the inner and the out core of each and every unique psyche (Patkar S V 2013) hence, psychiatry calls of personalized medicines which vary during the course of client's fluctuations in milieu exteriore, passages of life and evolution of defense mechanisms to cope with psychic repercussions to deal with distressed mind i.e. no target organ, it calls for holistic human concern.

In Psychiatry we see a plethora of symptoms manifested as behavioural anomalies, emotional aberration(s), and detoriation in already possessed personal capabilities or lack of maturation congruent with the passages of life on case to case basis. As a consequence of this phenomenon, each case needs to be taken up as a project, unique in itself, which necessitates personalized medicine in psychiatry. This calls for in depth understanding of the pharmacokinetics of each psychotropic agent we use, along with the pharmacogenetics of each subject (patient) which depends upon race, caste, creed, life style, food habits as well as genomorphics of the subject in hand. Haven't we seen patients who fail to respond to tricyclic agents, showing brilliant recovery with tetracyclics or showing brilliant response to piperazine, not with quinolinone derivatives, this bespeaks of psychotropic species impacting differentially on case to case basis. We have seen many cases of classical Manic Depressive Psychosis not adequately stabilized with adequate Lithium; Lamitrogine, but stabilized with Quietiapin; as also classical Schizophrenics with hallucinations, delusions, paranoid features not responding to Haloperidol, Resperidone, but brilliantly responding to Aripiprazole, iloperidone,Mirtazapine combination, these happening ought to make us aware that "personalized medicine is customized behavioural approach to management of health condition which calls for genetic understanding for difference in psycho pharmacotherapy i.e. pharmacogenomics (Downing Gregory J 2009)". This enables us to optimized individual factors to optimize or prevent psychiatric diseases. For personalized medication we need to know the Pharmacodynamics of the psychotropic agent we use, as well as the remediation of their effluvial effects.

Personalized medicine in psychiatry e.g. in the form of tailored psychotropic agents has already proved its efficacy, notably in terms of adjusted optimum doses and predictable drug responses or drug induced side effects (Evers Kathinka 2009). In order to understand the pharmacogenetics we need to understand as to how the psychiatric disorders operate at molecular level which entails tailoring choice as well as doses of the mind influencing agents we all use, which happens with psychotherapeutic bonding.

Psychotropic agents remain the cornerstone in treatment of psychiatric disorders. However, more than $20 \%$ of patients do not initially respond to treatment of drug therapy (Essali A, Al Haj, HN et al 2002). In addition to lack of response, many patients discontinue their medication due to side effects, which can have serious and devastating consequences ( Nose M et al 2003). The pharmacodynamics of drug metabolism depends upon Pharmacogenomics which calls for personalized psychiatric medication ( de Leon J. 2009) obtained from holistic intervention. Quality and Quantity of life depends upon junk gene expressing as white matter in brain tissue for which proper understanding of medication for expectations from a Psychiatrist is natural, as also possible, provided we amalgamate genetic adiposity versus life style adiposity, the former manifesting as HDL and latter LDL phenomenon which is in our domain of psychotropic intervention. Similarly, the electrical disturbance in heart are nerve conduction aberration phenomenon and need to be managed by us thereby preventing implantation of pace makers, as also is the case with essential hypertension managed at an early age for preventing Coronary Ischemic Disease and other systemic damage. Drugs affecting the hippocampus enhance grey matter. "The ultimate remedy lies in the development of social phenomenology infrastructure to handle their distribution, consolidation and prevention en-route personalized psychiatric medication, Cuijpers P 2009 has reviewed and found a growing number of randomized controlled trials 
have shown that it is possible at least in some cases to actually prevent or at lease delay the onset of mental disorder, including depressive and anxiety disorders and some studies indicate that it may be possible to prevent the onset of psychotic disorders in high risk group for which selecting target group by using indices other than odds ratios, relative risks or incidence rate ratios alone is insufficient". For studying the cumulative effect of joint exposures to several risk indicators rather than the effect of a single risk indicator for which shared decision making in mental health management is a necessity. Shared decision making, consists of a Psychiatrist with consultation liaison experience, a Clinical Psychologist, the patient himself and one of the care givers / family member ( Drake R. E. et al 2009), would assure personalized Psychiatric intervention impacting the Quality Of Life and enabling harnessing of full potential of an individual for meeting his realistic aspirations in life, which would not be otherwise possible.

\section{References}

1. Macher Jean Paul (2009): Dialogues in Clinical Neuro Science, 2009 Editorial, Volume 11, No. 4, pp.357.

2. Patkar S. V. (2013): Add grace to psychiatric practice, Indian J. Psychiatry. 2013, Vol. 55, No. 2 PP 197 - 199.

3. Downing J Gregory (2009): Policy perspectives on emerging pathways of personalized medicine: Dialogues in Clinical Neurosciences pp 379

4. Evers Kathinka (2009): Clinical research, Dialogues in Clinical Neurosciences PP. 432.

5. Essali A. Al Haj H. N. et al (2002): Clonazipine versus Typical Neuroleptic Medication for Schizophrenia (review). Cochrane Database Syst. Rev. 2002: D000059.

6. Nose M., Barbul C, Tanselta M (2002) How often do patients with psychosis fail to adhere to treatment programmes? A systematic review, Pshycho Med. 2003;331149- 1160.

7. de Leon J. (2009): Pharmacogenomics: the promise of personalized medicine for CNS disorder. Neuro Psychopharmacology, 2009, 34: 159-172.

8. Cuijpers Pim (2009): Prevention: an achievable goal in personalized medicine: Dialogues in Clinical Neurosciences; 2009, Volume 11, No. 4 pp, $447-454$.

9. Drake Robert E ; Cimpeon Delia; and Torrey William (2009): Shared decision making in mental health: prospects for personalized medicine. Dialogues in Clincal Neurosciences; 2009, Volume 11, No.4, pp 455-463.

\section{EDITOR}

Kumar Anil, MD (Psy.)

Consultant Psychiatrist

Santevita Hospital

Main Road, Ranchi - 83400

e-mail : anilsudesh1953@gmail.com

\section{CO-EDITOR}

S. R. P. Shukla MD (Psy) DPM (Psy)

Consultant Noetic Psychiatry

M - 61, Shyamli Colony

P. O. Doranda, Ranchi -834002

e-mail : govindshukla@msn.com

srpshukla@gmail.com 